\title{
Author Correction: Covidization of research: what are the risks?
}

\author{
Madhukar Pai (D)
}

Correction to: Nature Medicine https://doi.org/10.1038/s41591-020-1015-0, published online 27 July 2020.

In the version of this article initially published, the second sentence of the eighth paragraph failed to include the attribution for that text. The correct attribution is as follows: "As described by Ballantyne and Dunning (https://blogs.scientificamerican.com/observations/which-experts-should-you-listen-to-during-the-pandemic/\#: :text=In\%20philosophy\%2C\%20this\%20phenomenon\%20 has, where\%20they\%20lack\%20crucial\%20competence), by indulging in 'epistemic trespassing,' wherein well-intentioned scientists with real expertise in one field intrude into another...." The error has been corrected in the HTML and PDF versions of the article.

Published online: 16 October 2020

https://doi.org/10.1038/s41591-020-1127-6

๑ Springer Nature America, Inc. 2020

\section{Author Correction: A stochastic agent-based model of the SARS-CoV-2 epidemic in France}

Nicolas Hoertel (D), Martin Blachier, Carlos Blanco, Mark Olfson, Marc Massetti, Marina Sánchez Rico, Frédéric Limosin and Henri Leleu

Correction to: Nature Medicine https://doi.org/10.1038/s41591-020-1001-6, published online 14 July 2020.

In the version of this article initially published, two values in the final two sentences of the first paragraph of the 'Interventions' subsection of the Methods section (“...risk of transmission would be decreased by $53 \%$ if all individuals were wearing masks....both measures would reduce risk multiplicatively by $67 \%$...") were incorrect. The correct text is as follows: “...risk of transmission would be decreased by $47 \%$ if all individuals were wearing masks....both measures would reduce risk multiplicatively by $63 \%$...”. Also, four values in the 'Efficacy of masks' section of Supplementary Table 3 (top group, "If efficacy is fixed at 79\% (instead of 53\% in the main analysis)"; second group, "If efficacy is fixed at $36 \%$ (instead of $53 \%$ in the main analysis)") were incorrect. The correct values are as follows: "If efficacy is fixed at $64 \%$ (instead of $47 \%$ in the main analysis)" (top group); and "If efficacy is fixed at $21 \%$ (instead of $47 \%$ in the main analysis)" (second group). Finally, three values in the first sentence of the legend to Extended Data Fig. 4 (“...at $36 \%$ a, c, e, and $79 \%$ b, d, f, instead of $53 \%$...") were incorrect. The correct values are as follows: “...at $21 \% \mathbf{a}, \mathbf{c}, \mathbf{e}$, and $64 \% \mathbf{b}, \mathbf{d}, \mathbf{f}$, instead of $47 \%$.... The errors have been corrected in the HTML and PDF versions of the article.

Published online: 16 October 2020

https://doi.org/10.1038/s41591-020-1129-4

(C) The Author(s), under exclusive licence to Springer Nature America, Inc. 2020 